\title{
Beneficial Effects of Adding Topical Atorvastatin 5\% Cream to Topical Betamethasone 1\% Ointment on Chronic Hand Eczema
}

\author{
Maryam Mehrpooya, PhD'; Fatemeh Ghaed-Amini, PhD'; Farzin Firozian, PhD²; Younes Mohammadi, PhD³; Pedram Alirezaei, MD* \\ 'Department of Clinical Pharmacy, School of Pharmacy, Hamadan University of Medical Sciences, Hamadan, Iran \\ ${ }^{2}$ Department of Pharmaceutics, School of Pharmacy, Hamadan University of Medical Sciences, Hamadan, Iran \\ ${ }^{3}$ Modeling of Non-communicable Diseases Research Center, School of Public Health, Hamadan University of Medical Sciences, Hamadan, \\ Iran \\ ${ }^{4}$ Psoriasis Research Center, Hamadan University of Medical Sciences, Hamadan, Iran
}

\begin{abstract}
Background: Hand eczema (HE) refers to a common inflammatory dermatological condition. Several studies have shown that statins may have anti-inflammatory effects. This study aimed at investigating the efficacy of adding topical atorvastatin to topical betamethasone in the treatment of chronic HE.

Methods: This randomized, double-blind, placebo-controlled research was done between October 2017 and August 2018 in Hamadan, Iran. Of 130 cases treated for HE, 88 were randomly assigned to groups receiving either betamethasone $1 \%$ ointment plus atorvastatin $5 \%$ cream $(n=44)$ or betamethasone $1 \%$ ointment plus vehicle cream $(n=44)$. Both groups applied their medications twice a day for 10 days. The primary outcome was changes in the severity of $\mathrm{HE}$, assessed by hand eczema severity index (HECSI). The secondary outcomes were changes in itching evaluated via visual analogue scale (VAS) and quality of life examined through dermatology life quality index (DLQI).

Results: Seventy-two out of 88 eligible cases completed the study. The mean HECSI scores decreased in both groups after the intervention, although the change in HECSI was greater in the atorvastatin group (adjusted mean difference [AMD]: 5.756; 95\% $\mathrm{Cl}: 5.168$ to $6.344, P<0.001)$. The mean VAS scores decreased in both groups after the intervention, although the change in VAS was greater in the atorvastatin group (AMD: $10.535 ; 95 \% \mathrm{Cl}: 7.005$ to $14.064, P<0.001$ ). Treatment with topical atorvastatin was more effective in improving DLQI (AMD: $1.990 ; 95 \% \mathrm{Cl}: 1.821$ to $2.158, P<0.001$ ).

Conclusion: Addition of topical atorvastatin to topical betamethasone is beneficial in treatment of chronic HE.

Trial Registration: Identifier: IRCT2017070922965N10; https://www.irct.ir/.

Keywords: Anti-Inflammatory agents, Eczema, Statins

Cite this article as: Mehrpooya M, Ghaed-Amini F, Firozian F, Mohammadi Y, Alirezaei P. Beneficial effects of adding topical atorvastatin 5\% cream to topical betamethasone 1\% ointment on chronic hand eczema. Arch Iran Med. 2020;23(9):605-613. doi: 10.34172/aim.2020.71.
\end{abstract}

Received: September 9, 2019, Accepted: March 14, 2020, ePublished: September 1, 2020

\section{Introduction}

Hand eczema $(\mathrm{HE})$ refers to a common and often chronic skin disease. ${ }^{1}$ The lifetime prevalence of $\mathrm{HE}$, which is known to affect all age groups, is roughly estimated at $15 \%{ }^{2,3} \mathrm{HE}$ is clinically characterized by redness of skin, thickening of skin, scaling, edema, vesicles, hyperkeratotic areas, fissures, erosions, pruritus, and pain. The severity of $\mathrm{HE}$ ranges from very mild to severe, and even mild $\mathrm{HE}$ can have negative effects on the patient's quality of life (QoL). ${ }^{4}$ Despite its uncertain etiology, HE seems to be a multifactorial disorder. It is believed that the interaction between exogenous factors (e.g., skin allergens or irritants) and endogenous factors (e.g., atopy) contribute to HE development. ${ }^{5}$ Disruption of the stratum corneum structure or function is crucial for the HE etiopathogenesis. ${ }^{6}$

Disruption of the epidermal barrier may lead to the increased penetration of irritants through the skin, which is followed by local inflammation. ${ }^{7,8}$ Therefore, local inflammatory responses are vital for the development and maintenance of HE. Elevated expression of proinflammatory cytokines such as tumor necrosis factor alpha (TNF- $\alpha$ ), interleukin 1 (IL-1), IL-6, IL-8, IFN- $\gamma$ and IL-2, as well as granulocyte monocyte-colony stimulating factor has been observed in contact dermatitis. ${ }^{9,10}$ IFN- $\gamma$ may facilitate the adhesion of $\mathrm{T}$ cells to epidermal keratinocytes by enhancing the expression of adhesion molecules, as well as class I and II antigens. ${ }^{9}$ Moreover, it has been shown that IL-1 and IL-2 can trigger allergic contact dermatitis by inducing the proliferation of antigen-specific T-helper 1 cells. ${ }^{10}$

In view of their anti-inflammatory effects, topical corticosteroids are usually the mainstay of HE treatment. 
However, the applicability of topical corticosteroids is limited by the development of tachyphylaxis, as well as the increased risk of several adverse effects, such as skin atrophy, telangiectasia, and systemic absorption (in longterm use). ${ }^{11}$ As $\mathrm{HE}$ is usually chronic and recurrent, introduction of steroid-sparing agents can be an interesting therapeutic avenue for the treatment of HE.

Statins are competitive inhibitors of 3-hydroxy-3methylglutaryl-coenzymeA reductaseand havebeen applied extensively to treat atherosclerosis and cardiovascular diseases due to their lipid-lowering properties. ${ }^{12}$ Recently, accumulating evidence has demonstrated the antiinflammatory and immunomodulatory effects of statins. ${ }^{13}$ Consequently, their possible use in treating autoimmune diseases has been considered. Their beneficial role as adjunct therapy in various immunological disorders, like multiple sclerosis, rheumatoid arthritis, and systemic lupus erythematosus, supports their anti-inflammatory properties. ${ }^{11,14,15}$ Furthermore, in preliminary studies, the potential therapeutic value of statins against immunemediated dermatological diseases such as vitiligo and psoriasis has been investigated, and promising results have been reported. ${ }^{16}$ Some animal studies have also shown that topical statins may be able to control the symptoms of contact dermatitis. ${ }^{17}$

No clinical investigation has studied the efficacy of topical statins in the treatment of HE. Given the antiinflammatory effects of statins, in the current double-blind randomized controlled trial, we aimed at investigating the possible impact of topical atorvastatin on chronic HE.

\section{Materials and Methods}

Study Design

Patients with a diagnosis of chronic HE, referring to the Outpatient Dermatology Clinic of Hamadan University of Medical Sciences (HUMS, Hamadan, Iran) between October 2017 and August 2018, were enrolled in this double-blind, randomized, placebo-controlled trial. Our aim was to compare the clinical effects of applying betamethasone $1 \%$ ointment plus atorvastatin 5\% cream versus betamethasone $1 \%$ ointment plus vehicle cream in the treatment of chronic HE.

\section{Study Population and Randomization}

Patients were recruited in this double-blind, placebocontrolled parallel-group study if they met the following inclusion criteria at baseline: age between 18 and 65 years; clinical diagnosis of HE by an expert dermatologist; diagnosis of atopic eczema based on the Hanifin and Rajka's criteria; presence of $\mathrm{HE}$ for at least 6 months before referral to our clinic; HECSI (hand eczema severity index) score <40; presence of itching; not taking any topical or systemic glucocorticoids in the past 4 weeks; and not taking any topical or systemic anti-pruritic treatments one week before the study. On the other hand, the exclusion criteria at baseline and during the study were as follows: comorbid inflammatory diseases of the skin such as psoriasis; pregnancy, lactation, or anticipating pregnancy during the study; presence of lesions on any sites other than the hands; use of any medicines other than the prescribed medications during the study; any adverse effects leading to treatment intolerance; and poor adherence to treatment. Moreover, if there was any uncertainty about the diagnosis of hand lesion, the patient was excluded from the research.

Eligibility of 130 patients was assessed. However, 15 cases did not agree to participate in the study, and 27 subjects did not meet the inclusion criteria at baseline. The remaining 88 eligible subjects were allocated into either the control group $(n=44)$ or atorvastatin group $(n=44)$ via permuted block randomization (with four-patient blocks). An independent statistician was asked to generate the allocation sequence randomly by means of random number generator in Microsoft Excel 2016 software. The generated sequences were then placed in opaque, sealed, serially numbered envelopes and a person not involved with patient assessment or data analysis was asked to open the envelopes sequentially. Neither the physician nor the patients were aware of the group assignments.

\section{Study Interventions}

The patients were required to discontinue any topical or systemic medications for $\mathrm{HE}$ other than the emollients before entering the study (a four-week washout period). Both groups were treated with betamethasone 1\% ointment for 10 days (twice daily). Beside this treatment, patients in the atorvastatin group were instructed to use atorvastatin 5\% topical cream twice daily for 10 days, while patients in the control group were instructed to use the vehicle cream twice a day for the same duration. The patients were instructed to first apply the betamethasone ointment and then the vehicle or atorvastatin cream after 1 hour.

\section{Background Data}

Demographic variables (e.g. gender and age) were recorded. The patients were asked to provide information about their gender, chronological age (based on the calendar date on which they were born), and the duration of their HE (based on the date on which they first experienced the eczema symptoms and signs). To calculate BMI, the patients' weight and height were measured. We measured weight and height using a column scale with height rod (Seca, Hamburg, Germany). We measured weight to the nearest $100 \mathrm{~g}$ while the patient was in lightweight clothes and barefoot. Height was also measured barefoot to the nearest 0.5 centimeter. BMI was then determined by dividing weight $(\mathrm{kg})$ by height squared $\left(\mathrm{m}^{2}\right)$.

Outcome Variables

Our primary outcome was changes in the clinical severity 
of HE from baseline. The HE clinical severity was assessed in each case based on the HECSI score at baseline and 10 days after treatment. ${ }^{18}$ Generally, HECSI is a ranking instrument to assess the severity of $\mathrm{HE}$, with a score range of 0-360. In order to score the severity of HE by HECSI, each hand was divided into five areas, including the fingertips, fingers (excluding tips), palms, back of hands, and wrists. The intensity of six morphological signs, including erythema, induration/papulation, vesicles, fissures, scaling, and edema, was scored in each of the mentioned areas: 0 , no skin alterations; 1 , mild alterations; 2, moderate alterations; and 3, severe alterations. Moreover, the extent of involvement in each area was scored from 0 to $4(0,0 \% ; 1,1-25 \% ; 2,26-50 \% ; 3,51-75 \%$; and 4 , 76-100\%). To calculate the HECSI score, the score of the affected skin area was multiplied by the total sum of the intensity related to each morphological sign.

The intensity of itching as a secondary outcome was also recorded before and after treatment. A visual analogue scale (VAS) was used for scaling the severity of pruritus in each patient at baseline and after treatment. For this purpose, a 100-mm horizontal line was used, and cases were requested to rate the severity of pruritus from 0 (no pruritus) to 100 (the most severe pruritus they could imagine). ${ }^{19}$

Moreover, the impact of the skin disorder on the patient's QoL, as another secondary outcome, was investigated using the Persian version of Dermatology Life Quality Index (DLQI). The reliability and validity of DLQI in the Iranian population have been assessed by Aghaei and colleagues. ${ }^{20}$ This questionnaire includes 10 questions regarding the signs and feelings (questions 1 and 2), daily routine (questions 3 and 4), leisure activities (questions 5 and 6), and personal relationships (questions 8 and 9), as well as work and school (question 7) and treatment (question 10). ${ }^{20}$ To calculate the DLQI, the scores of these ten questions should be summed up, yielding a maximum score of 30 .

In addition, any adverse effects due to treatment were recorded. In order to evaluate the disadvantages of treatment, the subjects were asked to report any of the following symptoms: itching, burning sensation, redness, and exacerbation of symptoms if such symptoms were present prior to treatment. In addition, the type and severity (mild, moderate, and severe) of the adverse effects were recorded.

\section{Preparation and Stability of Atorvastatin 5\% Cream}

Atorvastatin 5\% topical cream (W/W) was prepared by mixing the aqueous phase containing polysorbate, cetyl alcohol, and distilled water in the organic phase consisting of glycerol monostearate, mineral oil, and benzoic acid. Also, $50 \mathrm{~g}$ of atorvastatin powder (Sobhan Darou, Iran) was added to the organic phase. The total weight of atorvastatin cream and vehicle cream was $100 \mathrm{~g}$. The selection of the $5 \%$ concentration of atorvastatin was based on a previous study conducted by Toker et al who used topical atorvastatin 5\% in the treatment of diabetic wounds. ${ }^{21}$ The stability of topical atorvastatin cream was evaluated by keeping the formulation in a germinator with $75 \%$ humidity at $60^{\circ} \mathrm{C}$ for six months. Stability of the topical cream was estimated at $99 \%$ at the end of the first month and $82.6 \%$ at the end of the sixth month.

To investigate the skin irritation potential of the atorvastatin cream, Draize rabbit skin irritancy test was conducted. ${ }^{22}$ To perform this test, the fur on the back of six healthy rabbits was shaved 24 hours before the application of the topical samples. The rabbit's back was divided into 4 marked areas $(25 \times 25 \mathrm{~mm})$ for the application of the atorvastatin cream, the base cream, distilled $\mathrm{H}_{2} \mathrm{O}$ (as negative control), and $15 \%$ sodium lauryl sulfate solution (as positive control). Both the treated and controlled sites were covered with a gauze patch for $24 \mathrm{~h}$. In 24,48 , and 72 hours after topical application, the sites were examined for skin irritation including erythema and edema. The severity of erythema and edema formation was scored from 0 (denoting no edema and erythema) to 4 (denoting severe edema and erythema). No dermal reactions including erythema or edema were observed at 24,48 , and 72 hours post-treatment in the rabbits. Thus, the topical formulation of atorvastatin was not irritant to the rabbit's skin and showed comparable tolerability to base cream.

\section{Sample Size Estimation and Statistical Methods}

Based on the findings of Ala et $\mathrm{al}^{23}$ a sample size of 74 patients (37 in each arm of the study) was calculated for detecting a minimally significant difference of 1.5 between the two groups in reducing the severity of $\mathrm{HE}$ (based on HECSI score), assuming a standard deviation of 1.9 for the treatment group and 2.25 for the placebo group with $80 \%$ power, $5 \%$ level of significance and a dropout rate of $20 \%$.

Demographic variables (e.g. age and gender), as well as BMI, eczema duration, HECSI, intensity of itching, and QoL (DLQI) were described at baseline. Categorical variables (e.g., gender) were reported as percentage. Means \pm standard deviations (SD) were also calculated for describing continuous variables. Assumption of normality was examined by Kolmogorov-Smirnov test. Independent samples $t$ test was applied for comparing independent quantitative variables with a normal distribution (age and BMI). In addition, chi-square test was employed to evaluate qualitative variables and Mann-Whitney $U$ test for comparing differences in variables with no normal distribution (HECSI, VAS, and DLQI) between the groups. Moreover, paired samples Wilcoxon test was performed for paired non-normally distributed data to determine changes in the outcome variables (HECSI, VAS and DLQI) before and after treatment. Analysis of covariance (ANCOVA) was applied to analyze the 
differences in severity of eczema (assessed by HECSI), intensity of itching (assessed by VAS) and QoL (assessed by DLQI) between the atorvastatin and control groups at the end of the trial, whilst adjusting for respective baseline values (baseline HECSI, baseline VAS and baseline DLQI).

Intention-to-treat analysis was performed to compare the participants within the groups. In this method, all patients who are randomized are analyzed according to their original group assignment, and those who violate the study protocol are not excluded from the statistical analysis. It is worth mentioning that the exclusion of those who violate the study protocol from the analysis may violate the principle of randomization, reduce the study power and exaggerate the treatment effect. ${ }^{24}$ Because of the dropout levels of smaller than $20 \%$ and same disease courses in both groups, missing values were replaced by the averages of the other group. ${ }^{25}$ Data analysis was performed using the SPSS 23 (IBM Corp., Armonk, NY, USA). P values smaller than 0.05 were regarded significant.

\section{Results}

Figure 1 presents the research flow diagram. Sixteen out of 88 eligible patients, who were divided into the atorvastatin and control groups via block randomization, did not complete the study due to the following reasons: intolerable adverse effects $(n=4)$; lost to follow-up $(n=6)$; and non-adherence to therapy $(n=6)$. Finally, 72 patients completed the study (37 patients in the atorvastatin group and 35 patients in the control group). Causes of patient loss during the study were comparable between the two groups $(P=0.790)$. As described in the statistical analysis section, intention-to-treat analysis was performed in this study.

Table 1 shows the subjects' demographics as well as baseline clinical characteristics. Overall, $69.4 \%$ of the participants were female. The atorvastatin and control groups showed no significant difference regarding age $(36.25 \pm 11.40$ versus $38.26 \pm 9.70$ years respectively, $P=$ $0.988)$. Moreover, Table 1 shows no significant difference among the atorvastatin and control groups regarding gender $(P=0.817)$. Similarly, no significant difference was found in BMI $\left(24.39 \pm 3.30 \mathrm{~kg} / \mathrm{m}^{2}\right.$ versus $23.28 \pm 3.20$ $\mathrm{kg} / \mathrm{m}^{2}$ respectively, $P=0.150$ ) and duration of eczema $(51.38 \pm 15.51$ versus $44.80 \pm 17.60$ months, respectively, $P=0.091)$.

Changes in the HECSI score during the study are shown in Table 2. The groups were not significantly different in terms of disease severity at baseline. The mean HECSI scores at baseline were $19.05 \pm 4.95$ and $19.14 \pm 5.22$ in the atorvastatin and control groups, respectively $(P=0.932)$. Analysis of HECSI scores showed that they decreased in both groups 10 days after treatment, although the change in the HECSI score was greater in the atorvastatin group $(10.66 \pm 2.59$ versus $4.93 \pm 1.17 ; P<0.001)$. In other words, improvement in the overall severity of HE (based on HECSI score) was greater in the atorvastatin group (Figure 2). Analysis of covariance (ANCOVA) also showed a statistically significant difference in post-intervention

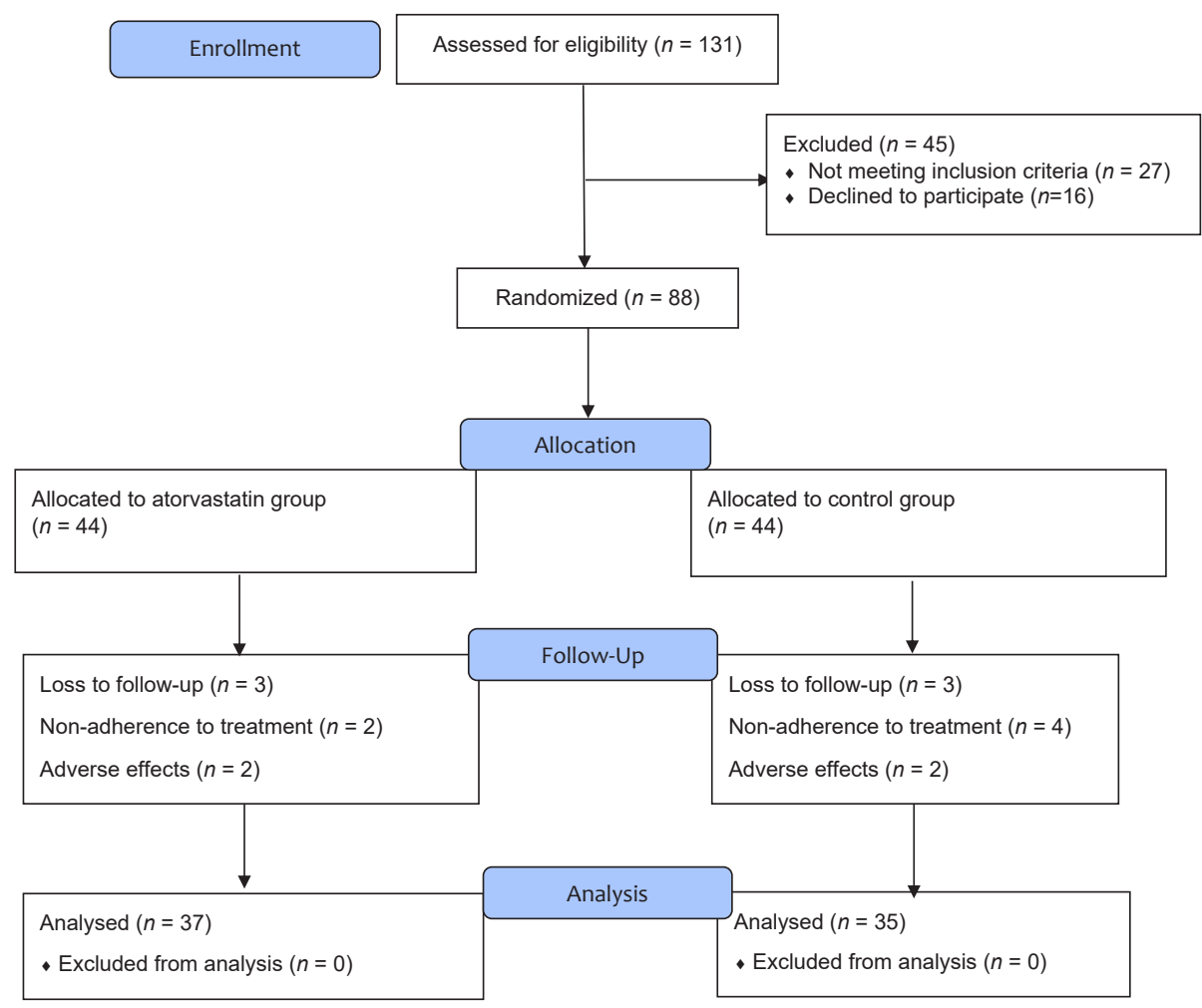

Figure 1. The Flowchart of the Study. 
Table 1. The Subjects' Characteristics at Baseline

\begin{tabular}{lccl}
\hline \multirow{2}{*}{ Variables } & \multicolumn{2}{c}{ Groups } & \\
\cline { 2 - 3 } & $\begin{array}{c}\text { Control } \\
(\mathbf{n}=\mathbf{4 4})\end{array}$ & $\begin{array}{c}\text { Atorvastatin } \\
(\mathbf{n}=\mathbf{4 4})\end{array}$ & $\boldsymbol{P}$ Value \\
\hline Gender (male/female) & $14 / 30$ & $13 / 31$ & 0.817 \\
\hline Age $(\mathrm{y})^{\mathrm{a}}$ & $38.26 \pm 9.7$ & $36.25 \pm 11.40$ & 0.988 \\
\hline $\mathrm{BMI}\left(\mathrm{kg} / \mathrm{m}^{2}\right)^{\mathrm{a}}$ & $23.28 \pm 3.2$ & $24.39 \pm 3.3$ & 0.150 \\
\hline Eczema duration (months) & $44.8 \pm 17.6$ & $51.38 \pm 15.51$ & 0.091 \\
\hline $\begin{array}{l}\text { BMl, body mass index. } \\
\text { a Values are expressed as mean } \pm \mathrm{SD} .\end{array}$ & & \\
\hline
\end{tabular}

A

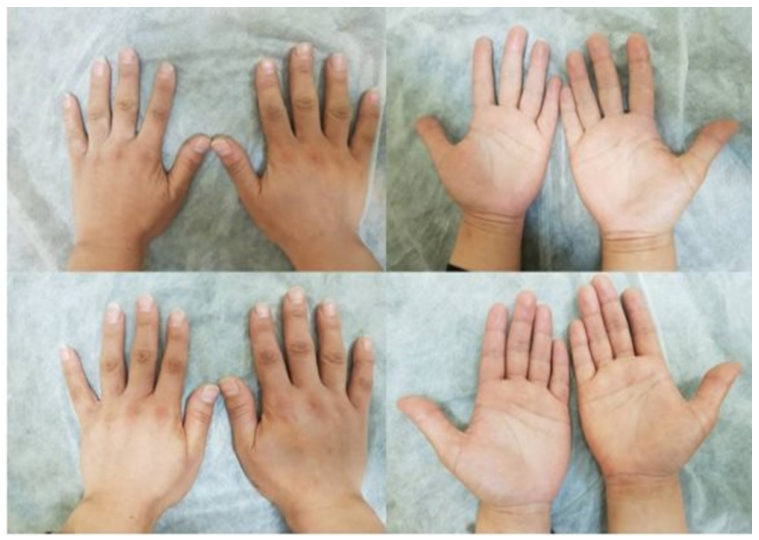

Figure 2. Image of Hands (A) before Treatment, (B) after 10 Days Treatment by Topical Atorvastatin and Betamethasone.

HECSI score (as dependent variable) between the atorvastatin group and the placebo group after controlling for pre-intervention HECSI score (as covariate) $(\mathrm{F}(1,84)$ $=378.873$, adjusted mean difference $[\mathrm{AMD}]=5.756$; $95 \%$ confidence interval for difference: 5.168 to 6.344 , $P<0.001)$.

Table 3 shows the intensity of itching in the two groups throughout the study based on VAS. No significant difference was found between the atorvastatin and control groups in VAS score at baseline $(56.45 \pm 22.07$ versus $56.73 \pm 21.19 ; P=0.887)$. While the intensity of itching decreased in both groups after 10 days of treatment, improvement in the mean VAS score from baseline was greater in the atorvastatin group than the control group
$(54.48 \pm 24.12$ versus $44.48 \pm 12.77 ; P=0.006)$. Analysis of covariance (ANCOVA) also showed a statistically significant difference in post-intervention VAS score (as dependent variable) between the atorvastatin group and placebo group after controlling for pre-intervention VAS score (as covariate) $(\mathrm{F}(1,84)=35.234$, AMD = 10.535; $95 \%$ confidence interval for difference: 7.005 to 14.064 , $P<0.001)$.

Table 4 shows the patients' QoL according to DLQI in the atorvastatin and control groups. No significant difference was observed between the atorvastatin and control groups regarding DLQI score at baseline (5.52 \pm 1.35 versus $5.55 \pm 1.42 ; P=0.973)$. Based on the results, while the total DLQI scores improved throughout the study in both groups, the mean change of DLQI score was significantly greater in the atorvastatin group than the control group $(2.62 \pm 0.79$ versus $0.49 \pm 0.71 ; P<0.001)$. ANCOVA also showed a statistically significant difference in post-intervention DLQI score (as dependent variable) between the atorvastatin group and placebo group after controlling for pre-intervention DLQI score (as covariate) $(\mathrm{F}(1,84)=552.200, \mathrm{AMD}=1.990 ; 95 \%$ confidence interval for difference: 1.821 to $2.158, P<0.001$ ).

Regarding the occurrence of adverse events, six patients $(16.2 \%)$ in the atorvastatin group and five $(14.2 \%)$ in the control group experienced adverse events, such as burning, erythema, and irritation. Adverse events were mild, and no systemic adverse reactions were observed. Two subjects in the atorvastatin group and two in the control group left the study due to adverse events. However, none of the events were serious or caused any complications for the patients. The two groups showed no significant difference regarding the occurrence of adverse events $(P=0.954)$.

\section{Discussion}

This research is the first randomized, double-blind, controlled clinical trial, assessing the effectiveness of topical statins in treatment of HE. Our findings showed that as an adjuvant therapy, atorvastatin cream can effectively ameliorate eczema severity and eczema-related pruritus. In addition, treatment with topical atorvastatin improved the

Table 2. Comparison of Eczema Severity Based on HECSI in the Atorvastatin and Control Groups (Mean \pm SD)

\begin{tabular}{|c|c|c|c|c|}
\hline \multirow{2}{*}{ HECSI Score } & \multicolumn{2}{|c|}{ Groups } & \multirow{2}{*}{$P$ value $(\mathrm{M}-\mathrm{W})^{*}$} & \multirow{2}{*}{$\begin{array}{l}\text { Difference of means } \pm \mathrm{SE} \\
(95 \% \mathrm{Cl})\end{array}$} \\
\hline & Control $(n=44)$ & Atorvastatin $(n=44)$ & & \\
\hline Baseline & $19.14 \pm 5.22$ & $19.05 \pm 4.95$ & \multirow{2}{*}{0.932} & \multirow{2}{*}{$\begin{array}{l}0.09 \pm 1.08 \\
(-2.07,2.25)\end{array}$} \\
\hline $95 \% \mathrm{Cl}$ & $(17.42,20.67)$ & $(17.54,20.55)$ & & \\
\hline Endpoint & $14.2 \pm 4.32$ & $8.39 \pm 3.22$ & \multirow{2}{*}{$<0.001$} & \multirow{2}{*}{$\begin{array}{l}5.82 \pm 0.81 \\
(4.20,7.43)\end{array}$} \\
\hline $95 \% \mathrm{Cl}$ & $(12.95,15.65)$ & $(7.57,9.52)$ & & \\
\hline Mean difference (baseline-endpoint) & $4.93 \pm 1.17$ & $10.66 \pm 2.59$ & \multirow{2}{*}{$<0.001$} & \multirow{2}{*}{$\begin{array}{l}5.73 \pm 0.43 \\
(4.87,6.58)\end{array}$} \\
\hline $95 \% \mathrm{Cl}$ & $(4.34,5.15)$ & $(9.71,11.29)$ & & \\
\hline$P$ value (Wilcoxon) ${ }^{* *}$ & $<0.001$ & $<0.001$ & & \\
\hline
\end{tabular}

HECSI, Hand Eczema Severity Index; Cl, confidence interval; SE, standard error.

$* P$ value of Mann-Whitney $U$ test for comparing HECSI between the two intervention groups.

${ }^{* *} P$ value of paired samples Wilcoxon test for comparing baseline HECSI with endpoint HECSI in each group. 
Table 3. Comparison of the Intensity of Itching Based on VAS in the Atorvastatin and Control Groups (mean \pm SD)

\begin{tabular}{|c|c|c|c|c|}
\hline \multirow{2}{*}{ HECSI Score } & \multicolumn{2}{|c|}{ Groups } & \multirow{2}{*}{$P$ Value $(\mathrm{M}-\mathrm{W})^{*}$} & \multirow{2}{*}{$\begin{array}{l}\text { Difference of Means } \pm \mathrm{SE} \\
\qquad(95 \% \mathrm{CI})\end{array}$} \\
\hline & Control $(n=44)$ & Atorvastatin $(n=44)$ & & \\
\hline Baseline & $56.73 \pm 21.19$ & $56.45 \pm 22.07$ & \multirow{2}{*}{0.887} & \multirow{2}{*}{$\begin{array}{c}0.27 \pm 4.61 \\
(-8.39,9.44)\end{array}$} \\
\hline $95 \% \mathrm{Cl}$ & $(49.43,62.61)$ & $(49.68,63.09)$ & & \\
\hline Endpoint & $12.25 \pm 9.91$ & $1.99 \pm 8.41$ & \multirow{2}{*}{$<0.001$} & \multirow{2}{*}{$\begin{array}{l}10.27 \pm 1.96 \\
(6.38,14.17)\end{array}$} \\
\hline $95 \% \mathrm{Cl}$ & $(9.36,15.53)$ & $(-0.58,4.53)$ & & \\
\hline Mean difference (baseline-endpoint) & $44.48 \pm 12.77$ & $54.48 \pm 24.12$ & \multirow{2}{*}{$<0.006$} & \multirow{2}{*}{$\begin{array}{l}10.00 \pm 4.11 \\
(1.82,18.18)\end{array}$} \\
\hline $95 \% \mathrm{Cl}$ & $(39.63,47.53)$ & $(49.52,62.94)$ & & \\
\hline$P$ value (Wilcoxon) $)^{* *}$ & $<0.001$ & $<0.001$ & - & - \\
\hline
\end{tabular}

VAS, visual analogue scale; $\mathrm{Cl}$, confidence interval; $\mathrm{SE}$, standard error.

$* P$ value of Mann-Whitney $U$ test for comparing VAS between the two intervention groups.

**P value of paired samples Wilcoxon test for comparing baseline VAS with endpoint VAS in each group.

Table 4. Comparison of Patients' Quality of Life According to DLQI in the Atorvastatin and Control Groups (Mean \pm SD)

\begin{tabular}{|c|c|c|c|c|}
\hline \multirow{2}{*}{ DLQI Score } & \multicolumn{2}{|c|}{ Groups } & \multirow{2}{*}{$P$ Value $(M-W)^{*}$} & \multirow{2}{*}{$\begin{array}{c}\text { Difference of Means } \pm \\
\text { SE }(95 \% \mathrm{Cl})\end{array}$} \\
\hline & Control $(n=44)$ & Atorvastatin $(n=44)$ & & \\
\hline Baseline & $5.55 \pm 1.42$ & $5.52 \pm 1.35$ & \multirow{2}{*}{0.973} & $0.02 \pm 0.29$ \\
\hline $95 \% \mathrm{Cl}$ & $(5.14,6.02)$ & $(5.11,5.93)$ & & $(-0.57,0.61)$ \\
\hline Endpoint & $4.73 \pm 1.17$ & $3.09 \pm 0.83$ & \multirow{2}{*}{$<0.001$} & $1.64 \pm 0.22$ \\
\hline $95 \% \mathrm{Cl}$ & $(4.38,5.07)$ & $(2.76,3.24)$ & & $(1.21,2.07)$ \\
\hline Mean Difference(baseline-endpoint) & $0.49 \pm 0.71$ & $2.62 \pm 0.79$ & \multirow{2}{*}{$<0.001$} & $1.61 \pm 0.18$ \\
\hline $95 \% \mathrm{Cl}$ & $(0.34,0.77)$ & $(2.30,2.75)$ & & $(1.25,1.98)$ \\
\hline$P$ value $(\text { Wilcoxon })^{* *}$ & $<0.001$ & $<0.001$ & - & - \\
\hline
\end{tabular}

DLQI, dermatology life quality index; $\mathrm{Cl}$, confidence interval; $\mathrm{SE}$, standard error.

*P value of Mann-Whitney $U$ test for comparing DLQI between the two intervention groups.

${ }^{* *} P$ value of paired samples Wilcoxon test for comparing baseline DLQI with endpoint DLQI in each group.

patients' QoL.

Eczema is a common inflammatory cutaneous disorder, with considerable detrimental effects on subjects' QoL. HE is probably one of the most common forms of eczema. ${ }^{26}$ Although the exact etiology of $\mathrm{HE}$ is not completely known, available evidence suggests an interaction between various genetic and environmental determinants. The increased allergens' penetration through the skin due to epidermal barrier dysfunction may lead to the activation of immune and inflammatory responses in the skin. ${ }^{11}$ Both innate and adaptive immune systems seem to be involved in this process.

Dysfunction of Toll-like receptors and high levels of proinflammatory cytokines (e.g., IL-4, IL-13, IL-31, and IL22) have been observed in skin lesions. ${ }^{27-30}$ Considering the crucial role of inflammatory processes in the pathogenesis of eczema, topical corticosteroids are broadly used as first-line treatment for HE. ${ }^{31-33}$ There are also other antiinflammatory drugs, such as topical calcineurin inhibitors, which can be used as steroid-sparing agents for severe or recalcitrant HE. ${ }^{34,35}$ However, the variability of patients' response to current treatment options, besides the development of several adverse events, especially due to long-term use of corticosteroids, has prompted researchers to seek alternative treatments.

Recent studies suggest that statins have potent antiinflammatory and immunomodulatory properties, independent of their effects on cholesterol. ${ }^{36}$ The exact mechanism through which statins exert their antiinflammatory effects is not fully recognized. Nevertheless, their inhibitory effects on a variety of immune responses such as lymphocyte migration and pro-inflammatory cytokine release may be responsible. ${ }^{37,38}$ Moreover, by blocking the synthesis of isoprenoid (a chemical activating inflammation via intracellular second messenger systems), statins may exert their immunomodulatory effects on innate and adaptive immune responses. ${ }^{38,39}$ The antipruritic properties of topical statins may be partly attributable to their immunomodulatory effects. In fact, it has been shown that proliferation of $\mathrm{T}$ lymphocytes and production of IL-2 may be decreased by statins. ${ }^{38,40}$ It is worth mentioning that IL-2 is one of the most important cytokines involved in itching. ${ }^{41}$ Furthermore, it has been shown that statins may have inhibitory impacts on nuclear factor Kappa B (NF-кB) activity, which in turn results in reduced expression of $\mathrm{T}$ helper 1 proinflammatory cytokines, such as IL-12 and TNF- $\alpha .{ }^{38}$ It has been shown that anti-inflammatory medications are capable of reducing pruritus, because they can control the inflammatory mechanisms responsible for the provocation of itching. ${ }^{41}$

According to the findings of the present study, statins can be potentially effective against inflammatory skin diseases. In previous studies, the potential therapeutic value of 
statins against various inflammatory disorders, including dermatological diseases, has been investigated. It has been shown that statins, particularly the simvastatin/ezetimibe combination, can effectively induce remission in patients with acute alopecia areata. Moreover, it has been shown that statins may be useful in preventing disease relapse. ${ }^{42}$ There are also some investigations assessing the potential application of statins against psoriasis. Some studies have reported that fluvastatin and simvastatin may have beneficial effects on psoriasis, ${ }^{43}$ while others have examined simvastatin alone and reported similar results. ${ }^{44,45}$ It seems that different statins, depending on their lipophilicity, may show different levels of anti-inflammatory properties. Therefore, the greater lipophilicity of fluvastatin, compared to other hydrophilic statins, may play a key role in its beneficial effects on psoriasis. ${ }^{46}$

Application of topical or systemic statins to treat acne vulgaris has yielded inconsistent results. Ahmadvand et al. indicated that the beneficial effects of oral and topical simvastatin, added to antibiotics, were superior to the use of antibiotics alone in patients with acne vulgaris. ${ }^{47}$ In contrast, Mikhael et al. demonstrated that topical atorvastatin might not be more effective than placebo when used as monotherapy for patients with papulopustular acne. ${ }^{48}$ It seems that combination of different topical statins may have more beneficial effects on acne lesions. Small sample size and short follow-up may be other drawbacks of the study conducted by Mikhael and colleagues. ${ }^{48}$

Recently, some investigations have examined the effectiveness of topical simvastatin in controlling contact dermatitis in animal models. A study by Otuki et al. showed that topical simvastatin might inhibit edema formation and migration of neutrophils in mice with contact dermatitis; this inhibitory effect was notably comparable to dexamethasone. ${ }^{49}$ Another study by Adami et al. investigated the potential beneficial effects of simvastatin $1 \%$ and simvastatin $3 \%$ on acute and chronic skin inflammation. Their findings revealed that in the acute type, both simvastatin $1 \%$ and $3 \%$ were able to reduce edema and migration of polymorphonuclear cells. However, in the chronic model, only simvastatin 1\% had positive effects on skin inflammation. ${ }^{50}$ It was found that chronic application of high concentrations of topical statins may result in skin barrier disruption and even skin inflammation. This is probably due to the reduced production of cholesterol by keratinocytes. ${ }^{51}$ Therefore, simultaneous application of cholesterol and ointments based on petrolatum and mineral oil is recommended to decrease the skin barrier damage caused by statins. ${ }^{52,53}$

Despite their beneficial effects on various dermatological diseases, statins seem to be responsible for several adverse effects on the skin or may even trigger inflammatory diseases such as psoriasis, systemic lupus erythematosus, dermatomyositis, and lichen planus. ${ }^{54-56}$ The preexisting skin barrier disruption, as well as genetic susceptibility, may be important factors in predisposing an individual to adverse cutaneous reactions to statins. ${ }^{57,58}$ Overall, statins may cause cutaneous adverse reactions, although they are very well-tolerated by patients; this can be best managed by discontinuation of the drug, which can result in rapid improvement of skin lesions in the majority of patients. ${ }^{57}$

Some limitations of this study might have affected the outcomes. The small sample size, short duration of the study, and exclusion of patients with severe $\mathrm{HE}$ are some of these limitations. However, it is worth mentioning that this trial was a pilot study to determine whether topical application of atorvastatin could have beneficial effects on chronic HE. Furthermore, despite the short duration of treatment, we could confirm the beneficial effects of topical atorvastatin on patients. Further studies with longer durations can be performed based on this pilot study. It should be also noted that we used atorvastatin as an adjunct therapy in the management of HE; therefore, the effectiveness of statins as monotherapy needs to be examined in future studies. Moreover, the topical atorvastatin formulation, which was used in the current study, is a basic formulation that definitely needs to be improved in future studies.

In conclusion despite the limitations of this study, our findings suggest that topical statins may have beneficial effects on chronic HE. Our findings also indicated that topical atorvastatin, as an adjunct therapy to topical corticosteroids, might be effective in improving the patients' QoL. According to the results, statins can be potential therapeutic options for the management of chronic HE. However, further studies are recommended to examine the efficacy of topical statins against other inflammatory skin conditions.

\section{Authors' Contribution}

MM: contributed to study design and drafting of the manuscript. FG, FF and PA: contributed to data collection and manuscript drafting. YM: contributed to study design and statistical analysis. MM and PA: supervised the study.

\section{Conflict of Interest Disclosures}

The authors declare no conflicts of interest.

\section{Ethical Statement}

Written informed consent was obtained from the all participants. The Ethics Committee of Hamadan University of Medical Sciences (Hamadan, Iran) approved the study protocol. The study was conducted in accordance with the Declaration of Helsinki. The study is registered at the Iranian Registry of Clinical Trials (identifier: IRCT2017070922965N10).

\section{Acknowledgements}

This study was based on the thesis of Fatemeh Ghaed-Amini (Thesis No. 9604132336) and performed under the supervision of the ViceChancellor for Research and Technology of Hamadan University of Medical Sciences, Hamadan, Iran.

\section{References}

1. Leung DY. Atopic dermatitis: new insights and opportunities 
for therapeutic intervention. J Allergy Clin Immunol. 2000;105(5):860-76. doi: 10.1067/mai.2000.106484.

2. Thyssen JP, Johansen JD, Linneberg A, Menné T. The epidemiology of hand eczema in the general populationprevalence and main findings. Contact Derm. 2010;62(2):7587. doi: 10.1111/j.1600-0536.2009.01669.x.

3. Johannisson A, Pontén A, Svensson $\AA$. Prevalence, incidence and predictive factors for hand eczema in young adults-a follow-up study. BMC dermatol. 2013;13(1):14. doi: 10.1186/1471-5945-13-14

4. Charan UP, Peter CD, Pulimood SA. Impact of hand eczema severity on quality of life. Indian Dermatol Online J. 2013;4(2):102-5. doi: 10.4103/2229-5178.110629.

5. Sehgal VN, Srivastava G, Aggarwal AK, Sharma AD. Hand dermatitis/eczema: current management strategy. J Dermatol. 2010;37(7):593-610. doi: 10.1111/j.13468138.2010.00845.x.

6. Elias PM, Schmuth M. Abnormal skin barrier in the etiopathogenesis of atopic dermatitis. Curr Allergy Asthma Rep. 2009;9(4):265-72. doi: 10.1007/s11882-009-0037-y.

7. Yokota $M$, Maibach HI. Moisturizer effect on irritant dermatitis: an overview. Contact Derm. 2006;55(2):65-72. doi: $\quad$ 10.1111/j.0105-1873.2006.00890.x.

8. Harding CR. The stratum corneum: structure and function in health and disease. Dermatol Ther. 2004;17:6-15. doi: 10.1111/j.1396-0296.2004.04s1001.x.

9. Thestrup-Pedersen K, Larsen CG, Rønnevig J. The immunology of contact dermatitis: a review with special reference to the pathophysiology of eczema. Contact Derm. 1989;20(2):8192. doi: 10.1111/j.1600-0536.1989.tb03113.x.

10. Agarwal US, Besarwal RK, Gupta R, Agarwal P, Napalia S. Hand eczema. Indian J Dermatol. 2014;59(3):213-24. doi: 10.4103/0019-5154.131372.

11. Bissonnette R, Diepgen TL, Elsner P, English J, GrahamBrown R, Homey B, et al. Redefining treatment options in chronic hand eczema (CHE). J Eur Acad Dermatol Venereol. 2010;24:1-20. doi: 10.1111/j.1468-3083.2010.03615.x.

12. Sirtori CR. The pharmacology of statins. Pharmacol Res. 2014;88:3-11. doi: 10.1016/j.phrs.2014.03.002.

13. Stoll LL, McCormick ML, Denning GM, Weintraub NL. Antioxidant effects of statins. Drug Today (Barc). 2004;40(12):975-90. doi: 10.1358/dot.2004.40.12.872573.

14. Lanzillo R, Orefice G, Quarantelli M, Rinaldi C, Prinster A, Ventrella $G$, et al. Atorvastatin combined to interferon to verify the efficacy (ACTIVE) in relapsing-remitting active multiple sclerosis patients: a longitudinal controlled trial of combination therapy. Mult Scler. 2010;16(4):450-4. doi: 10.1177/1352458509358909

15. Lv S, Liu Y, Zou Z, Li F, Zhao S, Shi R, et al. The impact of statins therapy on disease activity and inflammatory factor in patients with rheumatoid arthritis: a meta-analysis. Clin Exp Rheumatol. 2015;33(1):69-76.

16. Jowkar F, Namazi MR. Statins in dermatology. Int J Dermatol. 2010;49(11):1235-43. doi: 10.1111/j.13654632.2010.04579.x.

17. Sahebkar A, Rathouska J, Derosa G, Maffioli P, Nachtigal P. Statin impact on disease activity and C-reactive protein concentrations in systemic lupus erythematosus patients: a systematic review and meta-analysis of controlled trials. Autoimmun Rev. 2016;15(4):344-53. doi: 10.1016/j. autrev.2015.12.007.

18. Held E, Skoet R, Johansen J, Agner T. The hand eczema severity index (HECSI): a scoring system for clinical assessment of hand eczema. A study of inter-and intraobserver reliability. $\mathrm{Br} J$ Dermatol. 2005;152(2):302-7. doi: 10.1111/j.13652133.2004.06305.x.

19. Reich A, Heisig M, Phan NQ, Taneda K, Takamori K, Takeuchi
S, et al. Visual analogue scale: evaluation of the instrument for the assessment of pruritus. Acta Derm Venereol. 2012;92(5):497-501. doi: 10.2340/00015555-1265.

20. Aghaei S, Sodaifi M, Jafari P, Mazharinia N, Finlay AY. DLQI scores in vitiligo: reliability and validity of the Persian version. BMC Dermatol. 2004;4(1):8. doi: 10.1186/1471-5945-4-8.

21. Toker S, Gulcan E, Cayc MK, Olgun EG, Erbilen E, Ozay Y. Topical atorvastatin in the treatment of diabetic wounds. Am J Med Sci. 2009;338(3):201-4. doi: 10.1097/ MAJ.0b013e3181aaf209.

22. Draize JH, Woodard G, Calvery HO. Methods for the study of irritation and toxicity of substances applied topically to the skin and mucous membranes. J Pharmacol Exp Ther. 1944;82:377-90.

23. Ala S, Alvandipour $M$, Saeedi $M$, Hamidian $M$, Shiva A, Rahmani N, et al. Effects of topical atorvastatin (2\%) on posthemorrhoidectomy pain and wound healing: a randomized double-blind placebo-controlled clinical trial. World J Surg. 2017;41(2):596-602. doi: 10.1007/s00268-0163749-x.

24. Ranganathan P, Pramesh CS, Aggarwal R. Common pitfalls in statistical analysis: Intention-to-treat versus perprotocol analysis. Perspect Clin Res. 2016;7(3):144-6. doi: 10.4103/2229-3485.184823.

25. Unnebrink K, Windeler J . Intention-to-treat: methods for dealing with missing values in clinical trials of progressively deteriorating diseases. Stat Med. 2001;20(24):3931-46. doi: 10.1002/sim.1149.

26. Su JC, Kemp AS, Varigos GA, Nolan TM. Atopic eczema: its impact on the family and financial cost. Arch Dis Child. 1997;76(2):159-62. doi: 10.1136/adc.76.2.159.

27. Leung DY, Guttman-Yassky E. Deciphering the complexities of atopic dermatitis: shifting paradigms in treatment approaches. J Allergy Clin Immunol. 2014;134(4):769-79. doi: 10.1016/j. jaci.2014.08.008.

28. Kuo I-H, Carpenter-Mendini A, Yoshida T, McGirt LY, Ivanov $\mathrm{Al}$, Barnes $\mathrm{KC}$, et al. Activation of epidermal toll-like receptor 2 enhances tight junction function: implications for atopic dermatitis and skin barrier repair. J Invest Dermatol. 2013;133(4):988-98. doi: 10.1038/jid.2012.437.

29. Fallon PG, Sasaki T, Sandilands A, Campbell LE, Saunders SP, Mangan NE, et al. A homozygous frameshift mutation in the mouse Flg gene facilitates enhanced percutaneous allergen priming. Nat Genet. 2009;41(5):602. doi: 10.1038/ng.358.

30. Eyerich K, Pennino D, Scarponi C, Foerster S, Nasorri F, Behrendt $\mathrm{H}$, et al. IL-17 in atopic eczema: linking allergenspecific adaptive and microbial-triggered innate immune response. J Allergy Clin Immunol. 2009;123(1):59-66. doi: 10.1016/j.jaci.2008.10.031.

31. Bleeker J, Anagrius C, Iversen N, Stenberg B, Valentin KC. Double-blind comparative study of Corticoderm ${ }^{\circledR}$ cream+ Unguentum Merck $\AA$ and Betnovate $\AA$ cream+ Unguentum Merck ${ }^{\circledR}$ in hand dermatitis. J Dermatol Treat. 1989;1(2):8790. doi: 10.3109/09546638909086701.

32. Gupta AK, Shear NH, Lester RS, Baxter ML, Sauder DN. Betamethasone dipropionate polyacrylic film-forming lotion in the treatment of hand-dermatitis. Int J Dermatol. 1993;32(11):828-9. doi: $10.1111 /$ j.1365-4362.1993. tb02778.x.

33. Uggeldahl PE, Kero M, Ulshagen K, Solberg V. Comparative effect of desonide cream $0.1 \%$ and $0.05 \%$ in patients with hand eczema. Curr Therap Res. 1986;40(5):969-73.

34. Katsarou A, Makris M, Papagiannaki K, Lagogianni E, Tagka A, Kalogeromitros D. Tacrolimus $0.1 \%$ vs mometasone furoate topical treatment in allergic contact hand eczema: a prospective randomized clinical study. Eur J Dermatol. 2012;22(2):192-6. doi: 10.1684/ejd.2011.1615. 
35. Schliemann S, Kelterer D, Bauer A, John SM, Skudlik C, Schindera I, et al. Tacrolimus ointment in the treatment of occupationally induced chronic hand dermatitis. Contact Dermatitis. 2008;58(5):299-306. doi: 10.1111/j.16000536.2007.01314.x.

36. Tandon V, Bano G, Khajuria V, Parihar A, Gupta S. Pleiotropic effects of statins. Ind J Pharmacol. 2005;37(2):77-85. doi: 10.4103/0253-7613.15106.

37. Bu DX, Griffin G, Lichtman AH. Mechanisms for the anti-inflammatory effects of statins. Curr Opin Lipidol. 2011;22(3):165-70. doi: 10.1097/MOL.0b013e3283453e41.

38. Jasinska M, Owczarek J, Orszulak-Michalak D. Statins: a new insight into their mechanisms of action and consequent pleiotropic effects. Pharmacol Rep. 2007;59(5):483-99.

39. Weitz-Schmidt G. Statins as anti-inflammatory agents. Trends Pharmacol Sci. 2002;23(10):482-6. doi: 10.1016/s01656147(02)02077-1.

40. Kwak B, Mulhaupt F, Myit S, Mach F. Statins as a newly recognized type of immunomodulator. Nat Med. 2000; 6(12):1399-402. doi: 10.1038/82219.

41. Buddenkotte J, Steinhoff M. Pathophysiology and therapy of pruritus in allergic and atopic diseases. Allergy. 2010;65(7):805-21. doi: 10.1111/j.1398-9995.2010.01995.x.

42. Lattouf C, Jimenez JJ, Tosti A, Miteva M, Wikramanayake TC, Kittles C, et al. Treatment of alopecia areata with simvastatin/ ezetimibe. J Am Acad Dermatol. 2015;72(2):359-61. doi: 10.1016/j.jaad.2014.11.006.

43. Kim TG, Byamba D, Wu WH, Lee MG. Statins inhibit chemotactic interaction between CCL20 and CCR6 in vitro: possible relevance to psoriasis treatment. Exp Dermatol. 2011;20(10):855-7. doi: 10.1111/j.1600-0625.2011.01343.x.

44. Naseri M, Hadipour A, Sepaskhah M, Namazi M. The remarkable beneficial effect of adding oral simvastatin to topical betamethasone for treatment of psoriasis: a doubleblind, randomized, placebo-controlled study. Niger J Med. 2010;19(1):58-61. doi: 10.4314/njm.v19i1.54216.

45. Shirinsky IV, Shirinsky VS. Efficacy of simvastatin in plaque psoriasis: A pilot study. J Am Acad Dermatol. 2007;57(3):52931. doi: 10.1016/j.jaad.2007.05.040.

46. Qi XF, Kim DH, Yoon YS, Li JH, Jin D, Teng YC, et al. Fluvastatin inhibits expression of the chemokine MDC/CCL22 induced by interferon- $\boldsymbol{\gamma}$ in HaCaT cells, a human keratinocyte cell line.
Br J Pharmacol. 2009;157(8):1441-50. doi: 10.1111/j.14765381.2009.00311.x.

47. Ahmadvand A, Yazdanfar A, Yasrebifar F, Mohammadi Y Mahjoub R, Mehrpooya M. Evaluation the effects of oral and topical simvastatin as adjunct therapy in the treatment of acne vulgaris. Curr Clin Pharmacol. 2018;13(4):279-83. doi: 10.21 74/1574884713666180821143545.

48. Mikhael EM, Mohammad MY. Evaluation of the effect of topical atorvastatin solution for the treatment of papulopustular acne. Int J Curr Pharm Res. 2013;5(3):59-60.

49. Otuki MF, Pietrovski EF, Cabrini DA. Topical simvastatin: preclinical evidence for a treatment of skin inflammatory conditions. J Dermatol Sci. 2006;44(1):45-7. doi: 10.1016/j. jdermsci.2006.04.006.

50. Adami M, da Silveira Prudente A, Mendes DAGB, da Silva Horinouchi CD, Cabrini DA, Otuki MF. Simvastatin ointment, a new treatment for skin inflammatory conditions. J Dermatol Sci. 2012;66(2):127-35. doi: 10.1016/j.jdermsci.2012.02.015.

51. Mao-Qiang M, Feingold KR, Elias PM. Inhibition of cholesterol and sphingolipid synthesis causes paradoxical effects on permeability barrier homeostasis. J Invest Dermatol. 1993;101(2):185-90. doi: 10.1111/1523-1747.ep12363729.

52. Namazi M. Statins: novel additions to the dermatologic arsenal? Exp Dermatol. 2004;13(6):337-9. doi: 10.1111/j.09066705.2004.00208.x.

53. Lynde C. Moisturizers for the treatment of inflammatory skin conditions. J Drugs Dermatol. 2008;7(11):1038-43.

54. Dobritoiu AM, Forsea DG. Statins and the skin. Ther Pharmacol ClinToxicol. 2011;15:98-104.

55. Jacobi $\mathrm{T}$, Highet A. A clinical dilemma while treating hypercholesterolaemia in psoriasis. $\mathrm{Br} J$ Dermatol. 2003; 149(6):1305-6. doi: 10.1111/j.1365-2133.2003.05675.x.

56. Cozzani E, Scaparro M, Parodi A. A case of psoriasis worsened by atorvastatin. J Dermatol Case Rep. 2009;3(4):60-1. doi: 10.3315/jdcr.2009.1037.

57. Salna MP, Singer HM, Dana AN. Pravastatin-Induced Eczematous Eruption Mimicking Psoriasis. Case Rep Dermatol Med. 2017;2017:3418204. doi: 10.1155/2017/3418204.

58. Feldmann R, Mainetti C, SauratJ-H. Skin lesions due to treatment with simvastatin (Zocor $($ ). Dermatology.1993;186(4):272. doi: $10.1159 / 000247370$. 